\title{
Correlação clínica e radiológica de pacientes portadores de dor lombar crônica com a discografia
}

\author{
Clinical and radiological correlation in patients with low back pain \\ submitted a discography \\ Correlación de la clínica y radiología de pacientes portadores de \\ dolor lumbar crónico con la discografia
}

\author{
Emiliano Neves Vialle ${ }^{1}$ \\ Luiz Roberto Gomes Vialle' \\ Mauricio Santos Gusmão \\ Juan Esteban Suarez Henao ${ }^{3}$ \\ Túlio Albuquerque de Moura Rangel ${ }^{2}$ \\ Rubem Cardenas Moron²
}

\section{RESUMO}

Objetivo: correlacionar dados clínicos e de imagem com resposta à discografia em pacientes portadores de dor lombar crônica. Métodos: análise prospectiva de 33 discografias em 20 pacientes portadores de dor lombar crônica. A avaliação constou de exame clínico e radiológico por meio de um protocolo contendo dados da anamnese, VAS, Oswestry e achados da ressonância magnética. Resultados: dos 33 discos examinados, 14 exames foram positivos e 19 negativos. A média de idade dos pacientes com discografia positiva foi de 40,7 anos e para discografia negativa foi de 43,14 anos. Os pacientes com dor lombar associada à ciática tiveram discografia positiva em $87,5 \%$ dos casos, enquanto pacientes com apenas lombalgia, $50 \%$ $(\mathrm{p}=0,008)$. A positividade da discografia foi de $88,9 \%$ para pacientes com mais de quatro episódios, enquanto entre um a quatro episódios tiveram

\section{ABSTRACT}

Objective: to correlate discography findings with patients symptoms and imaging studies. Methods: thirty three discograms of 20 patients with chronic low back pain were prospectively evaluated comparing discography findings with clinical data (symptoms, VAS, Oswestry score) and imaging studies (MRI). Results: fourteen discograms were considered positive and 19 negative. The mean age for positive exams was 40.7 years, and on the negative, 43.1 years. Patients with back and leg pain had positive discograms on $87.5 \%$ of the cases, while patients with back pain, $50 \%(p=0.008)$. Discograms were positive in $88.9 \%$ of the patients that had had more than four acute episodes of pain, in comparison of $50 \%$ of those without acute episodes $(p=0.004)$. VAS and Oswestry score did not differ between groups; $80 \%$ of the patients who had HIZ on their MRI had positive discogram $(p=0.045)$, and $75 \%$
\end{abstract}

\section{RESUMEN}

Objetivo: correlacionar datos clínicos y de imagen con respuesta a la discografia en pacientes de dolor lumbar crónico. Métodos: análisis prospectiva de 33 discografias en 20 pacientes portadores de dolor lumbar crónico. La evaluación constó de un examen clínico y radiológico por medio de un protocolo que contiene datos de anamnesia, VAS, Oswestry y hallazgos de la RM. Resultados: de los 33 discos examinados, 14 exámenes fueron positivos y 19 negativos. El promedio de edad de los pacientes con discografia positiva fue de 40.7 años y para discografia negativa fue de 43.14 años. Los pacientes con dolor lumbar asociado a dolor del ciático tuvieron discografia positiva en $87.5 \%$ de los casos, mientras pacientes con apenas lumbalgia fueron un $50 \%(p=0.008)$. La positividad de la discografia fue de $88.9 \%$ para pacientes con mas de 4 episodios, mientras entre 1 a 4 episodios tuvieron positividad de $50 \%$ $(p=0.004)$. El EVA (Escala Visual Analó-

Trabalho realizado no Serviço de Ortopedia e Traumatologia do Hospital Universitário Cajuru da Pontifícia Universidade Católica do Paraná - PUCPR - Curitiba (PR), Brasil.

'Grupo de Cirurgia de Coluna do Hospital Universitário Cajuru da Pontifícia Universidade Católica do Paraná - PUCPR - Curitiba (PR), Brasil.

2Estagiários em Cirurgia de Coluna, AOSpine Latin America do Hospital Universitário Cajuru da Pontifícia Universidade Católica do Paraná - PUCPR - Curitiba (PR), Brasil.

${ }^{3}$ Residente do Serviço de Ortopedia e Traumatologia do Hospital Universitário Cajuru da Pontifícia Universidade Católica do Paraná - PUCPR - Curitiba (PR), Brasil. 
positividade de 50\% ( $\mathrm{p}=0,004)$. A Escala Visual Analógica (EVA) média e a avaliação conforme a escala de Oswestry não diferiram de modo significativo. Em relação à ressonância magnética, foi evidenciada uma positividade de $80 \%$ nos discos com HIZ (High Intesity Zone) $(\mathrm{p}=0,045) \mathrm{e}$ de $75 \%$ nos discos com alterações de $\operatorname{Modic}^{9}(p=0,083)$. O endpoint foi avaliado como com ou sem resistência á injeção intradiscal, e os exames com resistência tiveram menor positividade $(31,58 \%, p=0,0143)$. Conclusão: pacientes com queixa principal de dor lombar associada à ciática, que tiveram mais de quatroagudizações associadosa presença de HIZna ressonância mgnética têm maior incidência de positividade na discografia. Na discografia, endpoint com resistência tem maior associação com incidência de discos assintomáticos. Mesmo sem demonstrar diferença estatisticamente significante, existe uma forte associação entre alterações tipo Modic $^{9}$ com discografia positiva.

DESCRITORES: Dor lombar/ diagnóstico; Dor lombar/ radiografia; Disco intervertebral/radiografia; Imagem por ressonância magnética of those with Modic ${ }^{9}$ changes ( $p=0.083$ ).

Patients with a high resistance endpoint had few positive exams (31.58\%, $p=0.0143)$. Conclusion: patients with back and leg pain, with acute episodes, and HIZ on the MRI had a higher rate of positive discograms.

KEYWORDS: Low back pain/ diagnosis; Low back pain/ radiography; Intervertebral disc/radiography; Magnetic resonance imaging gica) promedio y la evaluación conforme la escala de Oswestry no difirió de modo significativo. En relación a la RM fue evidenciada una positividad de $80 \%$ en los discos con HIZ (High Intensity Zone) $(p=0.045)$ y de $75 \%$ en los discos con alteraciones de Modic ${ }^{9}(p=0.083)$. El endpoint fue evaluado con o sin resistencia a la inyección intradiscal y los exámenes con resistencia tuvieron menor positividad (31.58\%, p=0.0143). Conclusión: pacientes con queja principal de dolor lumbar asociado con dolor del ciático, que tuvieron más de cuatro agudizaciones asociadas a la presencia de HIZ en la RM tienen mayor incidencia de positividad en la discografia. En la discografia endpoint con resistencia tiene mayor asociación con la incidencia de discos asintomáticos. Aunque sin diferencia estadisticamente significativa, existe una fuerte asociación entre las alteraciones tipo Modic ${ }^{9}$ con discografia positiva.

DESCRIPTORES: Dolor de la región lumbar/diagnostico; Dolor de la región lumbar/ radiografia; Disco intervertebral/radiografia; Imagen por resonancia magnética

\section{INTRODUÇÃO}

A discografia foi inicialmente descrita por Lindblom ${ }^{1}$, em 1948, como uma punção seguida de injeção de contraste no disco intervertebral. Seu objetivo foi correlacionar os sintomas clínicos com os achados de imagem dos pacientes. Desde então, a discografia se tornou uma arma semiológica importante no diagnóstico e tratamento da dor lombar discogênica, principalmente pela baixa correlação entre as alterações degenerativas do disco intervertebral e a presença de sintomas. Estudos mostram que até um terço da população assintomática pode apresentar alterações discais degenerativas, na ressonância magnética $(\mathrm{RM})^{2}$.

A discografia gera discussões e polêmica, por ser um exame que necessita de informações do paciente e técnica correta, e sua sensibilidade diagnóstica foi motivo de inúmeros estudos. Holt3 realizou, em 1969, um estudo com discografia com voluntários jovens sadios, tentando realizar discografia em três níveis, e relatou
$37 \%$ de falsos positivos. Este estudo foi citado pelos que são contra a discografia, a ponto de que várias revisões foram publicadas a respeito, mostrando que Holt ${ }^{3}$ utilizou prisioneiros judiciários, que se beneficiavam da participação no estudo. Ainda, o estudo foi realizado sem radioscopia, com a punção dos discos sendo feita pela linha média (ou seja, transdural), com contraste que causava reação do sistema nervoso, para depois serem submetidos à radiografia controle. Carragee ${ }^{4}$ questionou a capacidade do paciente distinguir a dor causada pela discografia, das dores não axiais. Entretanto, Simmons ${ }^{5}$ obteve 81,4\% de acurácia e Walsh ${ }^{6}, 100 \%$ de sensibilidade considerando a discografia como positiva quando encontrava imagem anormal e dor importante.

A relação das anormalidades discais com os resultados da discografia também é motivo de controvérsia na literatura. Carragee ${ }^{7}$ evidenciou que não há diferença 
estatística em pacientes sintomáticos e assintomáticos com sinal de hiperintensidade (HIZ) na RM da coluna lombar submetidos à discografia. Entretanto, Schellhas ${ }^{8}$ relatou $87 \%$ de concordância em pacientes com HIZ lombar.

Apesar da discordância literária, os autores utilizam a discografia na rotina diária como teste semiológico e orientação do tratamento das doenças da coluna vertebral com o objetivo de determinar quais são suas relações clinicas e radiológicas.

Este estudo tem por objetivo correlacionar os achados da discografia com dados da história do paciente e de seus exames de imagem, a fim de identificar pacientes que não necessitariam da discografia lombar para definir a origem da dor.

\section{MÉTODOS}

Foi realizado um estudo prospectivo observacional no qual foram avaliadas 33 discografias em 20 pacientes com dor lombar refratária ao tratamento conservador (fisioterapia por seis meses), entre maio e novembro de 2006. Todos os exames foram realizados na coluna lombar entre L3 e S1 (Tabela 1). Todos os discos lombares degenerados de cada paciente foram estudados. Em um paciente foi realizado exame em três níveis degenerados, em 11 pacientes, dois níveis, e em oito pacientes, em apenas um nível, sempre com um nível normal utilizado como controle. A avaliação constou do preenchimento de consentimento informado, seguido de exame clínico e radiológico por meio de um protocolo contendo dados da anamnese (queixa, presença ou não de ciática, número de episódios), escala visual analógica da dor (EVA) diferenciando a dor lombar e dor ciática, questionário funcional (Oswestry) e da RM. Nesta última, o disco foi avaliado quanto à presença de degeneração, protrusão, hérnia, zona de hiperintensidade (HIZ), e alterações de Modic ${ }^{9}$.

Avaliação Clínica - Todos pacientes foram examinados pelos autores, sendo realizada anamnese e exame físico ortopédico completo. Por meio dos achados clínicos, foram submetidos à avaliação radiológica com radiografias e RM para hipótese diagnóstica de dor discogênica.

Achados Radiológicos - Como critérios de inclusão, foram avaliadas radiografias e RM. Pacientes com outras doenças que não degenerativas (alterações da estrutura óssea com espondilolistese, sequela de fratura) foram excluídos do estudo. Dentre os achados de RM, foi correlacionada presença de protrusão discal, hérnia segundo a Associação Americana de Radiologia, HIZ, alterações de Modic $^{9}$ ou apenas degeneração discal, com os resultados da discografia.

Discografia - Todas as discografias foram realizadas pelo autor principal sendo utilizada técnica com dupla agulha sob visão radioscópica, utilizando os critérios de Walsh et al. ${ }^{10}$ para determinar positividade do exame. Estes consistiam de dor à injeção de menos de $2 \mathrm{ml}$ de contraste, concordante à dor habitual do paciente, imagem de degeneração discal (contraste se espalha até a periferia do disco), e um disco controle (normal à RM) indolor.

Nos pacientes submetidos à discografia em mais de um nível, cefazolina (1g) foi misturada ao meio de contraste não-iônico como profilaxia de infecção ${ }^{11}$. Não foi utilizado nenhum tipo de sedação, a fim de permitir melhor interpretação da resposta dolorosa do paciente. Foi realizado novo EVA durante exame, para avaliar se a dor era concordante ou não, anotando volume de contraste injetado e se houve aumento da resistência à medida que o contraste era injetado (endpoint firme). A figura 1 mostra exemplo de imagens de uma discografia normal e uma anormal.

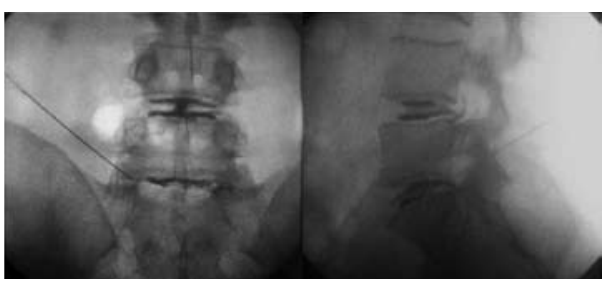

Figura 1

Exemplo de imagens de uma discografia normal e uma anormal

Avaliação Estatística - Os resultados obtidos foram tabulados em planilha do Microsoft Excel, e comparados com o teste do Qui-quadrado para dados não paramétricos.

\section{RESULTADOS}

Dentre os 33 exames realizados, 14 foram positivos e 19 negativos. Nenhum dos discos normais à ressância magnética, utilizados como controle, resultou em resposta dolorosa por parte do paciente. A média de idade dos pacientes com discografia positiva foi de 40,7 anos ( 25 a 56 anos) e para discografia negativa, 43,1 anos (30 e 55 anos). O sexo masculino teve positividade de $43,47 \%$, contra $40 \%$ no sexo feminino $(\mathrm{p}<0.05)$.

O nível L4-L5 foi o mais examinado (42,42\%), sendo a discografia positiva em $45,45 \%$ dos casos. Os outros níveis L2-L3, L3-L4 e L5-S1 corresponderam a 57,58\% dos casos (Tabela1).

Em relação à queixa principal, os pacientes com dor lombar associada à ciática tiveram discografia positiva em $87,5 \%$ dos casos, contra $50 \%$ dos casos de portadores de dor lombar pura $(\mathrm{p}=0,008)$ (Tabela 2$)$.

\section{TABELA 1 - Características dos pacientes e resultados da discografia}

\begin{tabular}{|c|c|c|}
\hline & $\begin{array}{c}\text { Discografia } \\
\text { positiva }(n=14)\end{array}$ & $\begin{array}{l}\text { Discografia } \\
\text { negativa }(n=19)\end{array}$ \\
\hline \multicolumn{3}{|l|}{ Média de idade } \\
\hline (geral - 41,55 anos) & $40,7(25-56)$ & $43,14(30-55)$ \\
\hline \multicolumn{3}{|l|}{ Sexo } \\
\hline masculino $(69,7 \%)$ & 10 & 13 \\
\hline feminino $(30,3 \%)$ & 4 & 6 \\
\hline \multicolumn{3}{|l|}{ Nível } \\
\hline L2-L3 (3,04\%) & 1 & - \\
\hline L3-L4 (18, 18\%) & 2 & 4 \\
\hline L4-L5 (42,42\%) & 5 & 9 \\
\hline L5-S1 $(36,36 \%)$ & 6 & 6 \\
\hline
\end{tabular}




\section{TABELA 2 - Correlação da queixa principal e resultado da discografia em 20 pacientes}

\begin{tabular}{lccc}
\hline Queixa principal & $\begin{array}{l}\text { Discografia } \\
\text { positiva }\end{array}$ & $\begin{array}{l}\text { Discografia } \\
\text { negativa }\end{array}$ & $\begin{array}{c}\text { Valor de } \\
\mathbf{p}\end{array}$ \\
\hline Lombalgia & $6(50 \%)$ & $6(50 \%)$ & NS \\
Lombalgia + ciática & $7(87,5 \%)$ & $1(12,5 \%)$ & $p=0,008$
\end{tabular}

Legenda: NS - não significante; $p>0,05$

TABELA 3 - Relação entre episódios prévios de dor e resultado da discografia

\begin{tabular}{|c|c|c|c|}
\hline Número de crises & Discografi & Discografia & Valor de \\
\hline de dor lombar & positiva & negativa & $p$ \\
\hline Nenhum & 1 & 0 & NS \\
\hline 1 a 4 & 5 & 5 & NS \\
\hline Mais de 4 episódios & 8 & 1 & $p=0,004$ \\
\hline
\end{tabular}

Legenda: NS - não significante; $p>0,05$

TABELA 4 - Relação da EVA e Oswestry com o resultado da discografia

\begin{tabular}{lccc}
\hline & $\begin{array}{l}\text { Discografia } \\
\text { positiva }\end{array}$ & $\begin{array}{l}\text { Discografia } \\
\text { negativa }\end{array}$ & $\begin{array}{c}\text { Valor de } \\
\mathbf{p}\end{array}$ \\
\hline EVA & $7,08(3-9)$ & $7,71(6-10)$ & $p=0,0078$ \\
Oswestry & $37,54(24-68)$ & $44,57(32-60)$ & NS \\
\hline
\end{tabular}

Legenda: NS - não significante; EVA- escala visual analógica; $p>0,05$
Quanto ao número de episódios de agudização de dor lombar, a incidência de positividade foi de $88,9 \%$ quando o paciente tinha mais de quatro episódios, enquanto entre um a quatro episódios tiveram positividade de $50 \%(\mathrm{p}=0,004)$ (Tabela 3).

O EVA médio na discografia positiva foi de 7,08 e, na negativa, $7,71(\mathrm{p}=0,0078)$. O Oswestry médio na discografia positiva foi de 37,53 e, na negativa 44,57 (Tabela 4).

Comparando-se as alterações da RM com o resultado da discografia, observamos que em $80 \%$ dos discos que apresentavam HIZ, a discografia foi positiva $(\mathrm{p}=0,045)$. Os discos que apresentavam alteraçoes de Modic (tanto I como II) foram positivos à discografia em $75 \%$ dos casos $(\mathrm{p}=0,083)$. Nos discos com degeneração discal (sem sinais de Modic ou HIZ), apenas houve uma incidência de positividade de só $14,3 \%(\mathrm{p}=0,157)$, nos discos com protrusão $50 \%(\mathrm{p}=0,083)$ e com hérnia discal central $66,7 \%$ ( $\mathrm{p}=$ 0,1572 ). Não houve falso-negativo neste estudo (Tabela 5). $\mathrm{Na}$ discografia, o EVA durante o exame das discografias positivas foi de 8,92 , enquanto que nas discografias negativas foi de $0,74(\mathrm{p}=0,0028)$. O volume médio de contraste injetado no disco foi de $1,75 \mathrm{ml}$ nas discografias positivas e de $2,18 \mathrm{ml}$ nas discografias negativas $(\mathrm{p}=0,1858)$. O endpoint foi avaliado como com ou sem resistência à injeção do contraste. Os exames com resistência tiveram menor incidência de discografias positivas $31,58 \%(\mathrm{p}=0,0143)$ (Tabela 6).

TABELA 5 - Relação entre achados da ressonância magnética e resultados da discografia

\begin{tabular}{lccc}
\hline Achados de ressonância magnética & Discografia positiva & Discografia negativa & Valor de $p$ \\
\hline Disco normal & 0 & 2 & NS \\
Apenas degeneração discal (disco negro) & 2 & 12 & NS \\
Degeneração + protrusão & 3 & 3 & $N S$ \\
Degeneração + hérnia & 2 & 1 & $N S$ \\
Degeneração + HIZ & 4 & 1 & $p=0,0455$ \\
Degeneração + alterações de Modic & 3 & 1 & $p=0,0832$ \\
\end{tabular}

Legenda: NS - não significante, HIZ - zona de hiperintensidade; $p>0,05$

TABELA 6 - Relação das características da discografia com os resultados do exame

\begin{tabular}{llll}
\hline & Discografia positiva & Discografia negativa & Valor de $p$ \\
\hline EVA durante exame & $8,92(8-10)$ & $0,74(2-0)$ & $\mathrm{p}=0,003$ \\
Volume injetado & $1,75 \mathrm{ml}(1-3)$ & $2,18 \mathrm{ml}(1,5-3)$ & $\mathrm{NS}$ \\
Resistência discal & & & \\
com resistência & $6(31,58 \%)$ & $13(68,42 \%)$ & $\mathrm{p}=0,014$ \\
sem resistência & $7(50 \%)$ & $7(50 \%)$ & $\mathrm{NS}$ \\
\hline
\end{tabular}

Legenda: NS - não significante, EVA - Escala Visual Analógica da dor; $p>0,05$ 


\section{DISCUSSÃO}

Desde a introdução da discografia como teste diagnóstico por Lindblom ${ }^{1}$, em 1948, muitos estudos foram publicados sobre a eficácia e importância deste exame na prática médica. Colhoun et al ${ }^{12}$, em 1988, publicaram um estudo com 162 pacientes, que utilizou a discografia como base para planejamento cirúrgico obtendo $89 \%$ de sucesso. Derby et $\mathrm{al}^{13}$, em 1999, evidenciaram em seu estudo que os pacientes com discografia positiva que foram submetidos à fusão circunferencial tiveram melhores resultados clínicos que os submetidos apenas à fusão posterior pela remoção discal e estabilização anterior. Baseados nestes e em outros estudos, os autores utilizam a discografia na rotina diária, sendo este exame de fundamental importância para indicação e planejamento cirúrgico.

Este estudo visou correlacionar achados clínicos e de imagem com o resultado da discografia, a fim de identificar quais pacientes teriam maior incidência de positividade. Dentre os dados clínicos, sexo, idade e nível acometido não tiveram relação de positividade com a discografia. Entretanto, a queixa principal e o número de episódios prévios de dor tiveram importante relação. Pacientes com queixa principal de lombalgia associada à ciática tiveram maior incidência de discografia positiva $87,5 \%$, que pacientes com apenas lombalgia $12,5 \%$ e com diferença estatisticamente significante.

Como mostra a Tabela 5, muitos pacientes não tinham achados de imagem que justificassem a ciática. Entretanto, tivemos uma alta taxa de discografia positiva em pacientes com lombalgia associada à ciática. Os autores creditam esses resultados ao fato da ressonância magnética ser um exame estático e realizado em decúbito dorsal que pode não identificar hérnias ocultas ou rupturas anulares, identificadas pela discografia.

Outro achado importante foi dos pacientes com mais que quatro episódios de agudizações, que tiveram 88,9\% de positividade, dado não encontrado em outros estudos na literatura. O EVA e Oswestry foram utilizados para graduar dor e incapacidade dos pacientes com dor lombar. Evidenciamos neste estudo que estes dados não tiveram relação com o resultado da discografia. Contudo, existem inúmeras causas de dor lombar que também promovem disfunção e muitas vezes maior que as de origem discogênica, que estão sendo avaliadas neste estudo.

A correlação entre a ressonância magnética e a discografia também foi motivo de muitos estudos. Dentre os achados avaliados, discos com HIZ e sinais de Modic foram os que tiveram maiores relações de positividade. Neste estudo, a presença de HIZ significou discografia positiva em $80 \%$ dos casos. Carragee et $\mathrm{al}^{7}$ publicaram um estudo que mostra não haver diferença estatística entre pacientes sintomáticos e assintomáticos com HIZ lombar submetidos à discografia. Schellhas et al 8 relataram $87 \%$ de concordância em pacientes com HIZ lombar, resultado similar ao do presente estudo. A presença de sinais de Modic teve positividade de $75 \%$. As alterações de Modic descritas em 1988 foram associadas à maior incidência de doença degenerativa discal e dor lombar ${ }^{14,15}$. Entretanto, apesar da forte associação com discografia positiva, não houve diferença estatística neste estudo.

Em relação aos dados da discografia, houve positividade ao exame com menor volume médio de contraste injetado no disco $1,75 \mathrm{ml}$ comparado a $2,18 \mathrm{ml}$ nas discografias negativas. O'Neill et al ${ }^{16}$ mostraram, em seu estudo de discografias com pressão controlada, que existe uma forte incidência de verdadeiros positivos quando há dor concordante ao injetar os primeiros mililitros de contraste no disco intervertebral. Nas discografias positivas, parece não haver necessidade de injetar mais contraste nos discos que apresentam sintomas com pequeno volume de contraste. Entretanto, nos discos assintomáticos, injeta-se um pouco mais de contraste para confirmar o exame como negativo. Por fim, o endpoint foi avaliado como com ou sem resistência à injeção do contraste no disco. Este estudo mostrou que pacientes com resistência discal preservada tiveram uma menor taxa de positividade $31,58 \%$ (comparado com pacientes sem resistência em que a taxa de positividade foi $50 \%$ ). Isso comprova que até mesmo discos com imagem radiológica de degeneração ainda podem manter suas propriedades estruturais, não necessitando intervenção cirúrgica $^{12,13}$.

\section{CONCLUSÃO}

Os autores concluem que pacientes com queixa principal de dor lombar associada à ciática, que tiveram mais de quatro agudizações de dor, com a presença de HIZ na ressonância magnética têm maior incidência de positividade na discografia. Apesar de não existir diferença estatisticamente significante, existe forte associação de alterações radiológicas tipo Modic com discografia positiva, sendo necessário estudo com maior número de pacientes para uma conclusão mais definitiva. Os autores não puderam encontrar sinais, tanto clínicos como radiográficos, que previssem o resultados da discografia. Esta segue como o único método para correlacionar os sintomas com as alteraçoes degenerativas da coluna lombar.

\section{REFERÊNCIAS}

1. Lindblom K. Diagnostic disc puncture of intervertebral discs in sciatica. Acta Orthop Scand 1948;17:231-239.

2. Boden SD, Davis DO, Dina TS, Patronal NJ, Wiesel SW: Abnormal magnetic-resonance scans of the lumbar spine in asymptomatic subjects. J Bone Joint Surg Am 1990;72:403-408.

3. Holt EP. The question of lumbar discography. J Bone Joint Surg Am 1968; 50:720-726.
4. Carregee EJ, Tanner CM, Yang B, Brito JL, Truong T: False-positive findings on lumbar discography: Reliability of subjective concordance assessment during provocative disc injection. Spine 1999;24:2542-2547. 
5. Simmons JW, Aprill CN, Dwyer AP, Brodsky AE: A reassessment of Holt's data on: "The question of lumbar discography". Clin Orthop 1988;237:120-124.

6. Walsh TR, Weinstein JN, Spratt KF, Lehmann TR, Aprill C, Sayre $\mathrm{H}$ : Lumbar discography in normal subjects. J Bone Joint Surg Am 1990;72:1081-1088.

7. Carragee EJ, Tanner CM, Khurana $\mathrm{S}$, et al: The rates of false-positive lumbar discography in select patients without low back symptoms. Spine 2000;25:1373-1381.

8. Schellhas KP, Pollei SR, Gundry CR, et al. Lumbar disc high-intensity zone: Correlation of magnetic resonance imaging and discography. Spine 1996;21:79-86.

9. Modic MT, Steinberg PM, Ross JS, Masaryk TJ, Carter JR. Degenerative disk disease: assessment of changes in intervertebral body marrow with MR imaging. Radiology, 166: 193-199, 1988.

10. Walsh T, Weinstein J, Spratt K, et al. Lumbar discography in normal subjects: A controlled prospective study. J Bone Joint Surg [Am] 1990;72:1081-8.
11. Osti OL, Fraser RD, Vernon-Roberts B. Discitis after discography. The role of prophylactic antibiotics. J Bone Joint Surg Br. 1990 Mar;72(2):271-4.

12.Calhoun E, Mccall IW, Williams L, Cassar Pullicino VN: Provocation discography as a guide to planning operations on the spine. J Bone Joint Surg 1988;70:267-71.

13.Derby R, Howard MW, Grant JM, et al. The ability of pressure-controlled discography to predict surgical and nonsurgical outcomes. Spine 1999;24:364-71.

14.Kjaer P, Korsholm L, Bendix T, Sorensen JS, Leboeuf-Yde C.: Modic changes - their associations with other lumbar MRI findings and their attribution to low back pain. Eur Spine J (2006) 15:1312-1319.

15. Weishaupt D, Zanetti M, Hodler J, Min K, Fuchs B, Pfirmann CW, Boos N. Painful Lumbar Disk Derangement: Relevance of Endplate Abnormalities at MR Imaging. Radiology 2001; 218:420-427.

16.O’Neill C, Kurgansky M. Subgroups of Positive Discs on Discography. Spine 2004;29:2134-9.

\section{Correspondência}

Emiliano Neves Vialle

Rua Brig ${ }^{\circ}$ Franco, n 979

CEP: 80430-210

Tel.: + 5541 3223-7860

E-mail: emiliano@vialle.com.br 